Article

\title{
Tunable Goos-Hänchen Shift Surface Plasmon Resonance Sensor Based on Graphene-hBN Heterostructure
}

\author{
Zihao Liu, Fangyuan Lu, Leyong Jiang ${ }^{\mathbb{D}}$, Wei Lin and Zhiwei Zheng * \\ School of Physics and Electronics, Hunan Normal University, Changsha 410081, China; \\ liuzihao0514@hunnu.edu.cn (Z.L.); 201830133040@smail.hunnu.edu.cn (F.L.); \\ jiangly28@hunnu.edu.cn (L.J.); linweihbhg@hunnu.edu.cn (W.L.) \\ * Correspondence: zhengzhiwei@hunnu.edu.cn
}

\section{check for}

updates

Citation: Liu, Z.; Lu, F.; Jiang, L.; Lin, W.; Zheng, Z. Tunable Goos-Hänchen Shift Surface Plasmon Resonance Sensor Based on Graphene-hBN Heterostructure. Biosensors 2021, 11, 201. https://doi.org/10.3390/ bios11060201

Received: 9 May 2021

Accepted: 15 June 2021

Published: 21 June 2021

Publisher's Note: MDPI stays neutral with regard to jurisdictional claims in published maps and institutional affiliations.

Copyright: (c) 2021 by the authors. Licensee MDPI, Basel, Switzerland. This article is an open access article distributed under the terms and conditions of the Creative Commons Attribution (CC BY) license (https:// creativecommons.org/licenses/by/ $4.0 /)$.

\begin{abstract}
In this paper, a bimetallic sensor based on graphene-hexagonal boron nitride (hBN) heterostructure is theoretically studied. The sensitivity of the sensor can be improved by enhancing the Goos-Hänchen $(\mathrm{GH})$ shift in the infrared band. The theoretical results show that adjusting the Fermi level, the number of graphene layers and the thickness of hBN, a GH shift of $182.09 \lambda$ can be obtained. Moreover, sensitivity of $2.02 \times 10^{5} \lambda / R I U$ can be achieved with monolayer graphene, the thickness of gold layer is $20 \mathrm{~nm}$, silver layer is $15 \mathrm{~nm}$, and the hBN thickness of $492 \mathrm{~nm}$. This heterogeneous infrared sensor has the advantages of high sensitivity and strong stability. The research results will provide a theoretical basis for the design of a new high-sensitivity infrared band sensor.
\end{abstract}

Keywords: Goos-Hänchen (GH) shift; graphene; sensor

\section{Introduction}

Goos-Hänchen (GH) shift refers to the slight lateral deviation of the actual reflected beam relative to the geometrical optics predicted beam when the beam is reflected at the interface between the media. Since it was demonstrated by Goos and Hänchen in 1947 [1], GH shift has been widely studied in optical waveguide switch [2], narrow band optical filter [3], high sensitivity temperature sensor [4] and other fields. However, usually, the GH shift of the optical band is only in the order of wavelength, which makes it difficult to be measured in experiments, which hinders its practical application to a certain extent. In the past decade, the rapid development of nano-machining technology has set off a research boom of various new types of artificial microstructure materials. Thus, a diverse variety of microstructures has been designed by researchers to amplify GH shift. For example, negative refractive index materials [5], weak absorption medium dielectric slab [6], symmetrical metal-cladding optical waveguide [7] and periodic structure [8], photonic crystal [9], etc. However, after the internal parameters of these traditional structures are fixed, it is difficult to achieve passive regulation. This makes flexible regulation of $\mathrm{GH}$ displacement based on new semiconductor materials combined with traditional structures become a new research direction.

In recent years, two-dimensional materials represented by graphene and molybdenum disulfide $\left(\mathrm{MoS}_{2}\right)$ have excellent optoelectronic characteristics such as high electron mobility and adjustable external field [10-12]. Jiang et al. studied the GH shift of the reflected light beam on the graphene and realized the positive and negative conversion of the GH shift under the action of electronic control [13]. Tang et al. achieved a tunable GH shift in the terahertz band by utilizing magneto-optical effects in graphene [14]. It can be found from many cases of regulating GH shift that the material characteristics and structural parameters can have a significant impact on GH shift, so it is a good method to realize sensing performance by monitoring GH shift signal. Based on this idea, Zhou et al. designed a refractive index sensor with adjustable sensitivity coefficient by precisely controlling the GH shift in the graphene-substrate system at the $1550 \mathrm{~nm}$ optical communication band. By 
tuning the Fermi energy, the sensitivity coefficient can reach $\pm 1 \times 10^{6}$ [15]. Also, You et al. proposed that based on the graphene-MoS 2 heterostructure, under the action of two $\mathrm{MoS}_{2}$ layers and three layers of graphene at the $632.8 \mathrm{~nm}$ band, the maximum GH shift is obtained and the optimum sensitivity of $5.545 \times 10^{5} \lambda$ is achieved [16]. Therefore, the application of two-dimensional materials to the sensor in GH shift interrogation mode can not only obtain large GH shifts, but also realize the flexible adjustment of sensitivity coefficient.

In addition, improving sensor sensitivity has always been the goal of researchers. Traditional types of sensors usually use Au and Ag to improve sensor sensitivity [17]. For example, at $633 \mathrm{~nm}$ wavelength, Shushama et al. designed a refractive index sensor in angular interrogation mode by combining a two-dimensional material with gold. The proposed sensor has a maximum sensitivity of $210^{\circ} / R I U$ [18]. Fouad et al. achieved a high sensitivity of $280^{\circ} / R I U$ using an $\mathrm{Ag}-\mathrm{BaTiO}_{3}$ sensor at $632.8 \mathrm{~nm}$ [19]. However, these sensors also have some shortcomings. On the one hand, due to the poor binding ability of Au with biomolecules, it is difficult to improve the sensitivity of the sensors. On the other hand, although pure silver can provide a higher sensitivity, the pure silver exposed in the air oxidizes easily and has poor chemical stability [20]. So, researchers are considering combining gold and silver as bimetallic sensors to improve the chemical stability and sensitivity of the device. Although higher sensitivity can be provided by pure silver, it is easy to oxidize and has poor chemical stability. Therefore, the bimetallic sensors composed of gold and silver are used to improve the chemical stability and sensitivity of the devices. Since its more useful optical properties than pure gold or silver, sensor based on bimetal have also attracted a lot of attention [21,22]. Such as Liu et al. reported a novel biosensor in angular interrogation mode, which has an $\mathrm{Ag} / \mathrm{Au} / \mathrm{BaTiO}_{3} /$ graphene bimetallic configuration at the wavelength of $633 \mathrm{~nm}$. Sensitivities as high as $294^{\circ} / R I U$ were obtained by optimizing the parameters [23]. The success of these cases also provides a way to improve the sensitivity of the sensor in GH shift interrogation mode. However, in general, researchers mostly consider improving the sensitivity of the sensor in the optical band, and the infrared band is an important band of solar radiation, so it is more valuable to apply the composite structure to the infrared band. It is worth mentioning that hexagonal boron nitride (hBN), as a natural hyperbolic material, is often used as a substrate material and a dielectric material for high-performance devices in the infrared band. Due to its unique phonon characteristics, the graphene-hBN heterostructure has exhibited many excellent optical properties in the infrared band [24]. For example, in the infrared band, a graphene-hBN heterostructure is used to improve the reflected group delay [25], achieve perfect absorption [26], and enhance near-field thermal radiation [27].

Inspired by the above discussion, it has been shown that a bimetal composite structure based on graphene-hBN can achieve a large GH shift and high-sensitivity sensors. A giant GH shift was obtained by optimizing the Fermi energy, the number of graphene layers, and the hBN thickness. In addition, our structure can also be used as a sensitivity-adjustable refractive index sensor based on GH shift modulation to obtain the best sensitivity up to $2.02 \times 10^{5} \lambda / R I U$.

\section{Theoretical Model}

The analysis structure of K-R is shown in Figure 1, which includes BK7 prism, Ag-Au bimetallic film, hBN, graphene, and sensing layer. When a transverse magnetic (TM)polarized beam incident on BK7 prism, the evanescent-wave propagates along the prism metal interface and penetrates the metal layer. Once resonance occurs at a certain angle (resonance angle $\theta$ ), the minimum reflectivity can be obtained. Thus, the phase mutation will be caused, and a large GH shift will be produced. 


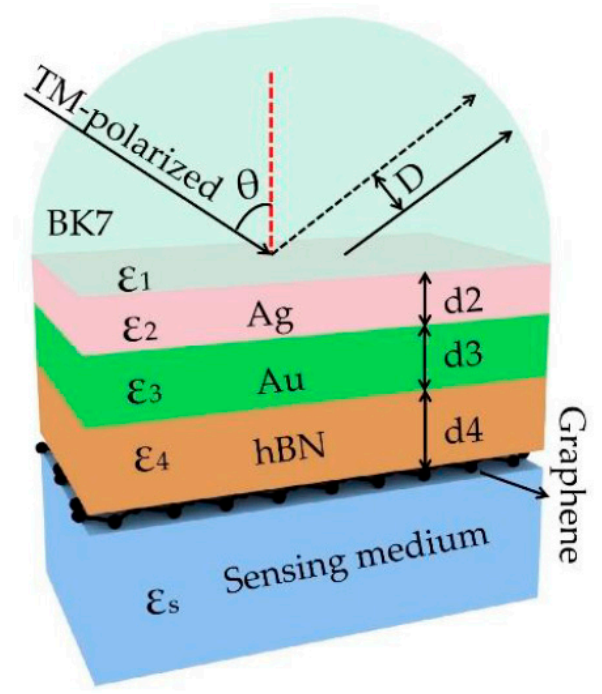

Figure 1. A bimetal structure based on graphene-hexagonal boron nitride (graphene-hBN) of schematic diagram.

In this device, the dielectric constant of BK7 is given by the relationship [28]

$$
\varepsilon_{1}=\frac{1.03961212 \lambda^{2}}{\lambda^{2}-0.00600069867}+\frac{0.231792344 \lambda^{2}}{\lambda^{2}-0.0200179144}+\frac{1.01046945 \lambda^{2}}{\lambda^{2}-103.560653}+1,
$$

where $\lambda$ is the incident wavelength and the unit is $\mu \mathrm{m}$. According to the Drude-Lorentz model, the dielectric constant of the metal layer is [29]

$$
\varepsilon_{m}=1-\frac{\lambda^{2} \lambda_{c}}{\lambda_{p}^{2}\left(\lambda_{c}+i \lambda\right)}
$$

Among them, $m=2,3$. Here $\lambda_{p}=1.4541 \times 10^{-7} \mathrm{~m}$ and $\lambda_{c}=1.7614 \times 10^{-5} \mathrm{~m}$ correspond to the collision and plasma wavelengths of the $\mathrm{Ag}$, respectively. For $\mathrm{Au}, \lambda_{p}=1.6826 \times 10^{-7} \mathrm{~m}$ and $\lambda_{c}=8.9324 \times 10^{-6} \mathrm{~m}$.

hBN has two infrared different reststrahlen active optical phonon modes in the infrared band. Its dielectric constant can be expressed as [26]

$$
\varepsilon_{u}=\varepsilon_{\infty, u}\left(1+\frac{\omega_{L O, u}^{2}-\omega_{T O, u}^{2}}{\omega_{T O, u}^{2}-\omega^{2}-i \omega \gamma_{u}}\right)
$$

In the formula, $u=x, y$ represents the transverse direction $(a, b$ axis direction of crystal face), $u=z$ represents the $z$ axis ( $c$ axis direction of crystal lattice). $\varepsilon_{\infty}$ and $\gamma$ represent the high frequency dielectric constant and attenuation constant respectively. All data parameters here are taken from the literature [26].

The surface conductivity $\sigma$ of graphene can be described in Kubo's form. Without considering the external magnetic field, the surface conductivity $\sigma$ of graphene can be expressed as the sum of in band conductivity and inter band conductivity [30]

$$
\begin{gathered}
\sigma_{\text {intra }}(\omega)=\frac{e^{2} K_{B} T}{\pi h^{2}} \frac{i}{\omega+i \tau_{g}^{-1}}\left[\frac{E_{F}}{K_{B} T}+2 \ln \left(e^{-\frac{E_{F}}{K_{B} T}}+1\right)\right], \\
\sigma_{\text {inter }}(\omega)=\frac{i e^{2}}{4 \pi h^{2}} \ln \left[\frac{2 E_{F}-\left(\omega+i \tau_{g}^{-1}\right) h}{2 E_{F}+\left(\omega+i \tau_{g}^{-1}\right) h}\right]
\end{gathered}
$$


Here, $\omega$ is the angular frequency of the incident light, $e$ and $h$ are the electron charge and the reduced Planck constant, respectively. $E_{F}$ and $K_{B}$ are Fermi energy and Boltzmann constants, respectively. $T$ is the temperature in $K$ and we set it to $300 \mathrm{~K}$. Moreover, $\tau_{g}$ is the relaxation time of graphene.

The dielectric constant of the sensing layer can be expressed as

$$
\varepsilon_{s}=n_{s}^{2}=\left(1.33+\Delta n_{s}\right)^{2}
$$

where $\Delta n_{s}$ is the amount of changes in refractive index of the sensing medium.

For multilayer structures, we can use the improved transfer matrix method to analyze the reflection phase $\left(\varphi_{r}\right)$ and reflectivity $\left(R_{r}\right)$. The transfer matrix of the composite structure can be expressed as [25]

$$
M=D_{B K 7 \rightarrow A g} P_{A g} D_{A g \rightarrow A u} P_{A u} D_{A u \rightarrow h B N} P_{h B N} D_{\text {Sensing }}
$$

Here, $D$ represents the transfer matrix at the interface, and $P$ is the propagation matrix in the medium. Then we can get $r=M_{21} / M_{11}$, where $r$ is the reflected coefficient of the structure. At the same time, the corresponding reflectance $R_{r}=|r|^{2}$ and reflected phase $\varphi_{r}(\theta, \omega)$ can be obtained by reflection coefficient. For the incident beam with sufficient waist, the GH shift of the reflected beam can be calculated by the steady-state phase method [12]

$$
D_{G H}(\theta, \omega)=-\frac{\lambda}{2 \pi} \frac{d \varphi_{r}}{d \theta}=-\frac{\lambda}{2 \pi} \frac{1}{|r|^{2}}\left(\operatorname{Re}(r) \frac{d \operatorname{Im}(r)}{d \theta}-\operatorname{Im}(r) \frac{d \operatorname{Re}(r)}{d \theta}\right),
$$

where $\lambda$ is the corresponding incident wavelength.

The sensor of the GH shift interrogation mode analyzes the sensing performance by detecting the GH shift. Therefore, the sensitivity of the sensor can be defined as [16]

$$
S=\frac{\Delta D_{G H}}{\Delta n_{S}}
$$

Here, $\Delta D_{G H}$ is defined as the amount of change in GH shift caused by changes in the refractive index of the sensing layer, and $\Delta n_{s}=0.005$.

\section{Results and Discussion}

Since the refractive index of the sensing layer of composite structure has a significant impact on the GH shift, the sensor based on GH shift interrogation mode can achieve the sensor performance by monitoring the GH shift signal. However, if the GH shift is too small, it may lead to errors in the monitoring results or not get better sensitivity, which will affect the application of refractive index sensor in real life. There are some important parameters of the composite structure that affect the GH shift, such as Fermi energy, the number of layers of graphene, thickness of hBN, and thickness of bimetal. The optimal sensitivity of the sensor can be obtained by finding a group of optimal structural parameters. Here, it is assumed that the incident wavelength of TM-polarized beam is $\lambda=10.176 \mu \mathrm{m}$. Also, the corresponding parameters are gradually optimized to improve the sensitivity of the structure.

First of all, the influence of Fermi energy on the reflectance, reflected phase and GH shift can be plotted and the corresponding curve is drawn in Figure 2. It can be seen from Figure $2 \mathrm{a}$ that there is an obvious dip of the reflectance near the resonance angle. The dip is caused by the excitation of surface plasmon polariton (SPP) on the surface of the metal medium when resonance occurs. Therefore, the power of the incident light is mostly absorbed and transferred to the SPP wave so that the reflectance curve drops sharply at the resonance angle. As the Fermi energy increases, the reflectance curve moves to higher angle. Then, the reflected phase curve also has an obvious mutation near the 
resonance angle. What's more, the slopes of reflection phase curves are different near the resonance angle at different Fermi energy, which can be found in Figure 2b. It is worth noting that we know from Formula (8) that in order to obtain a large GH displacement, a large slope of the reflection phase is required. Therefore, by applying the gate voltage to tune the Fermi energy of graphene to alter it from $0.1 \mathrm{eV}$ to $0.4 \mathrm{eV}$, it can be clearly seen from Figure $2 b$ that the slope of the reflected phase first increases and then decreases. When $E_{F}=0.2 \mathrm{eV}$, the reflected phase curve is the steepest. More importantly, two obvious features of GH shift can be found in Figure 2c. On the one hand, with the increase of Fermi energy, GH shift shows a tendency of increasing first and then decreasing. On the other hand, the corresponding GH shift is affected by reflection phases with negative slopes and shows positive values. At the same time, the reflected phase changes dramatically near the resonance angle, which makes the maximum GH shift value obtained. Especially, when the $E_{F}=0.2 \mathrm{eV}$, the normalized GH shift of $182.09 \lambda$ can be obtained near the resonance angle. These phenomena further confirm the validity of regulating GH shift by voltage. This provides an effective way to enhance the GH shift flexibly and obtain the optimum sensitivity of the structure.

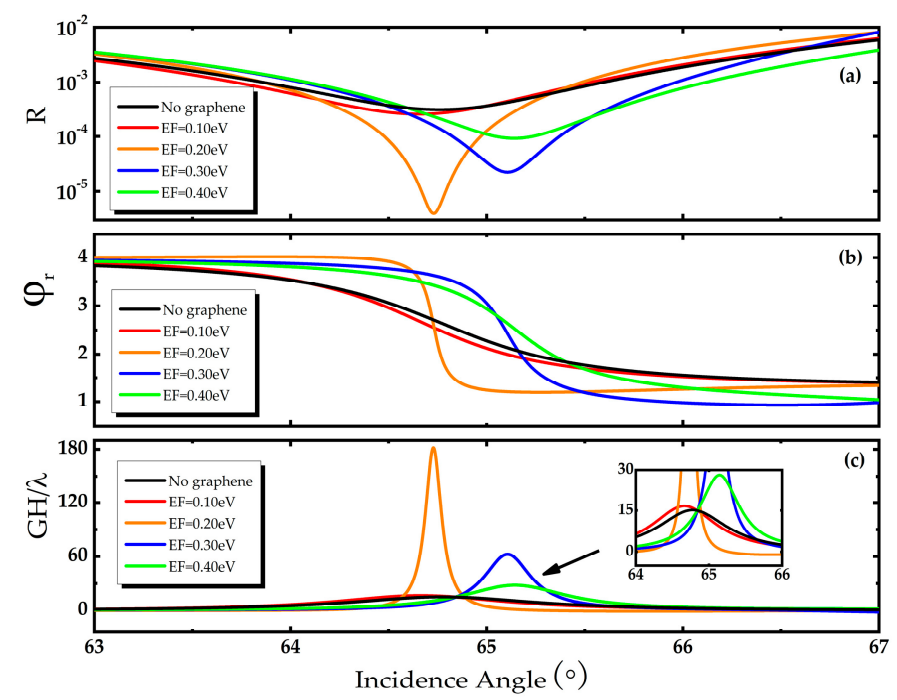

Figure 2. (a) Reflectance $R$ as a function of incident angle at different Fermi energy, (b) Reflected phase $\varphi_{r}$ as a function of incident angle at different Fermi energy, (c) Goos-Hänchen (GH) shift as a function of incident angle at different Fermi energy, the inset is an enlarged view of the GH shift in the range of $64 \sim 66^{\circ}$, in which Ag thickness $d_{2}=15 \mathrm{~nm}$, Au thickness $d_{3}=20 \mathrm{~nm}$, hBN thickness $d_{4}=131 \mathrm{~nm}$, graphene layer $N=1$.

Note that for graphene with a small number of layers $(N<6$, where $N$ is the number of layers), the optical conductivity of graphene with a small number of layers can be approximately expressed as $N \sigma$ if only considering the graphene sheets of each single atomic layer are non-contact [13]. Under this assumption, it is convenient to discuss the effect of the number of graphene layers on the GH shift, and the results are shown in Figure 3. On the whole, when the graphene layer is a single layer, the dip of reflectance is the deepest, and the reflection phase is the steepest, thus a large GH shift of $182.09 \lambda$ is obtained. Then, as the number of graphene layers increases, the reflectance in Figure $3 a$ is enhanced and the dip becomes shallow. Also, the slope of the reflection phase is decreasing (see Figure $3 b$ ) which leads to a corresponding reduce of the GH shift. Fortunately, when graphene does not exist, there is a GH shift of about $15 \lambda$ in this composite structure. As mentioned above, if the sensitivity of the sensor is to be improved, the GH shift of the structure should be enhanced first, because this can make the detection of GH shift signal more accurate and convenient, and the sensitivity of the sensor can be improved correspondingly. It can be known from Figure 3 that the GH shift obtained when monolayer 
graphene is selected for calculation is the largest, so monolayer graphene is selected for the sensor sensitivity research in the following research.

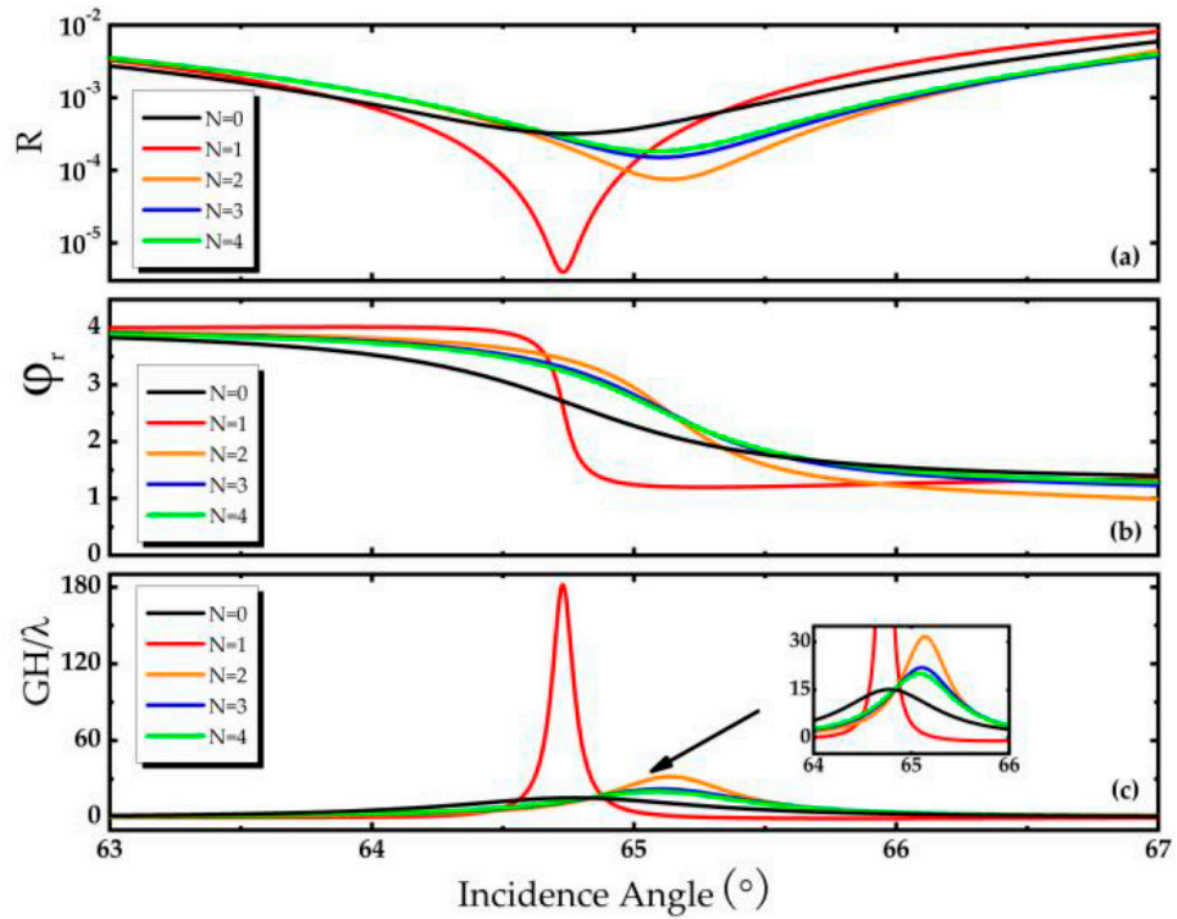

Figure 3. (a) Reflectance $R$ as a function of incident angle at different layers of graphene, (b) Reflected phase $\varphi_{r}$ as a function of incident angle at different layers of graphene, (c) GH shift as a function of incident angle at different layers of graphene, in which Fermi energy $E_{F}=0.2 \mathrm{eV}$, Ag thickness $d_{2}=15 \mathrm{~nm}$, Au thickness $d_{3}=20 \mathrm{~nm}, \mathrm{hBN}$ thickness $d_{4}=131 \mathrm{~nm}$.

At the same time, the thickness of $\mathrm{hBN}$ is also an important control parameter in increasing GH shift. Not only does it have Lorenz resonance characteristics in the infrared band, but it is also an important factor in the propagation matrix. Therefore, a curve is drawn in Figure 4, which enhances GH shift and improve the sensitivity of the structure by selecting the appropriate thickness. It can be found in the figure that the GH shift value increases initially then reduces, with the enlargement of hBN thickness (in a small range). In addition, the maximum $\mathrm{GH}$ shift keeps rising in a single period. When the hBN thickness is $492 \mathrm{~nm}$, the GH shift reaches a normalized shift value of 920.66. Furthermore, in the illustration of Figure 4, the sensitivity curve is drawn with the thickness of hBN is $492 \mathrm{~nm}$. Also, the sensitivity can be reached $3.18 \times 10^{4} \lambda / R I U$ when the angle is $64.68^{\circ}$. However, the thickness of the material is too large, which is not conducive to the fabrication of ultra-thin refractive index sensor and may have some defects. So, in this paper, for the convenience of research, we choose the thickness of hBN as $131 \mathrm{~nm}$, and the GH shift value obtained here is $182.09 \lambda$. 


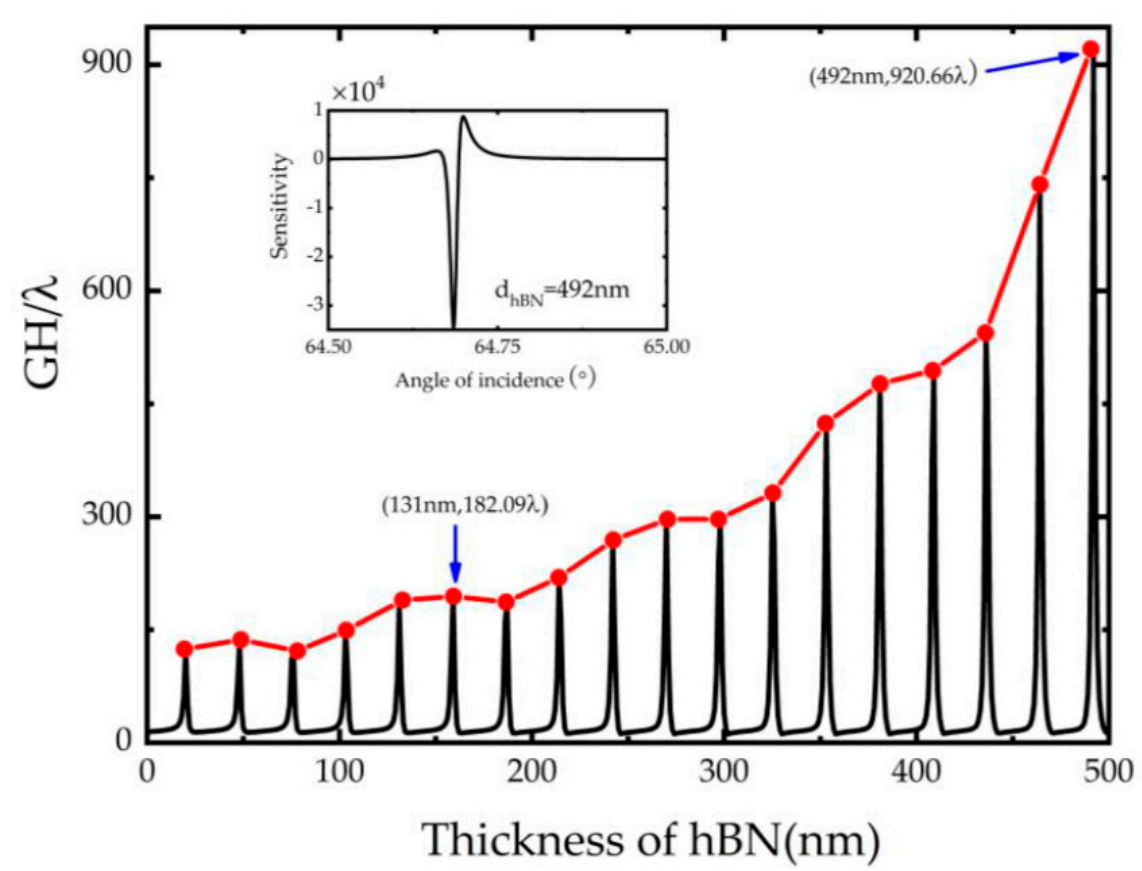

Figure 4. Dependence of GH shift on the thickness of hBN, in which Fermi energy $E_{F}=0.2 \mathrm{eV}, \mathrm{Ag}$ thickness $d_{2}=15 \mathrm{~nm}$, Au thickness $d_{3}=20 \mathrm{~nm}$, graphene layer $N=1$ and the inset is the sensitivity curve at $d_{3}=492 \mathrm{~nm}$.

We know that Ag is the most suitable material for providing high sensitivity, while $\mathrm{Au}$ has great advantages in excellent oxidation resistance and better chemical stability. Therefore, it is necessary to select the thickness of each layer reasonably for obtaining higher sensitivity and stability. In Table 1, we show the best sensitivity under different thickness of gold and silver. In general, when Au is constant, the sensitivity increases first and then decreases with the rising of Ag. Also, when Ag stays the same, the sensitivity increases initially then reduces with the enlargement of Au. More importantly, when the thickness of the gold layer is $20 \mathrm{~nm}$ and the thickness of the silver layer is $15 \mathrm{~nm}$, the maximum sensitivity $(2083 \lambda / R I U)$ can be obtained at $\theta=64.72^{\circ}$ by calculation. Therefore, the Au thickness of $20 \mathrm{~nm}$ and silver thickness of $15 \mathrm{~nm}$ are considered in the study of the composite structure, which is more convenient to obtain the ideal sensitivity.

Table 1. Optimum sensitivity $(\lambda / R I U)$ for different gold and silver thicknesses.

\begin{tabular}{ccccccc}
\hline $\mathbf{A u} / \mathbf{A g}(\mathbf{n m})$ & $\mathbf{1 4}$ & $\mathbf{1 5}$ & $\mathbf{1 6}$ & $\mathbf{1 7}$ & $\mathbf{1 8}$ & $\mathbf{1 9}$ \\
\hline 16 & 0.2747 & 0.3625 & 0.5110 & 0.8081 & 1.6794 & 29.3081 \\
18 & 0.6258 & 1.0099 & 2.2402 & 777.6916 & 21.4966 & 4.7777 \\
20 & 5.4571 & 2083 & 92.2965 & 7.7814 & 3.0161 & 2.7537 \\
22 & 3.8497 & 8.1241 & 45.6784 & 437.8950 & 143.1114 & 21.2400 \\
24 & 0.7618 & 0.7794 & 1.0741 & 1.3421 & 1.5868 & 1.9990 \\
\hline
\end{tabular}

In the previous discussion, we mentioned that Fermi energy has a great influence on GH shift and obtains a large GH shift $(182.09 \lambda)$ at $E_{F}=0.2 \mathrm{eV}$. Here, we will consider the sensitivity regulation of Fermi energy when the composite structure is applied to the sensor. In Figure 5, the sensitivity curve is plotted when the Fermi energy changes from 0.1 to $0.4 \mathrm{eV}$. With the increase of Fermi energy, the optimal sensitivity increases first and then decreases. Obviously, the trend of sensitivity with Fermi energy is consistent with the trend of GH. When the Fermi energy is $0.2 \mathrm{eV}$, the best sensitivity is obtained under the current parameter. It is further proved that enhancing GH shift value is an effective way to improve sensitivity. 

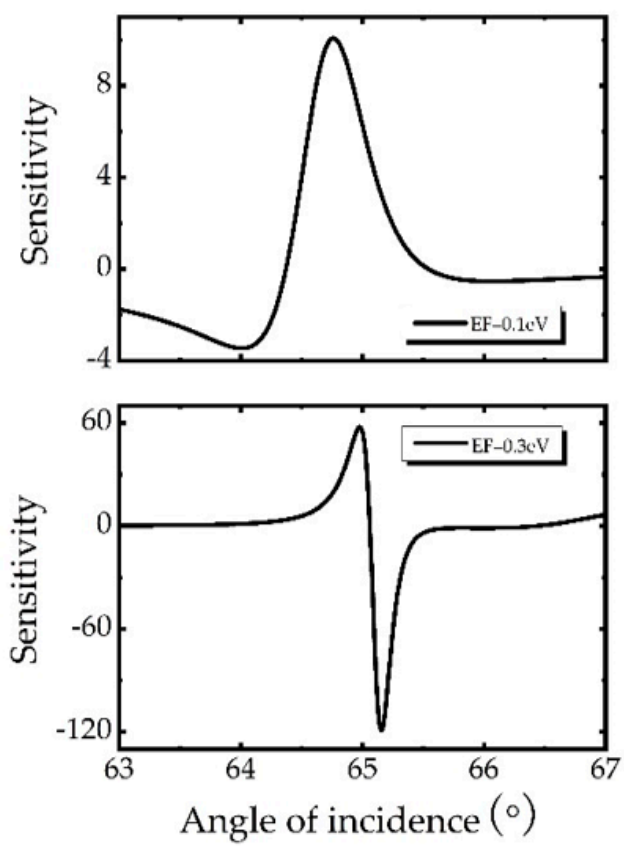
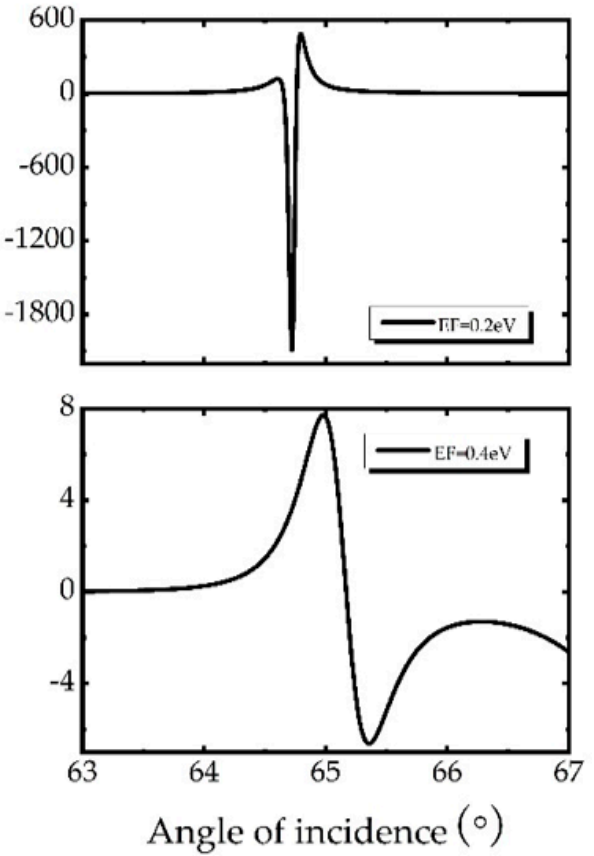

Figure 5. Dependence of sensitivity on Fermi energy, in which Ag thickness $d_{2}=15 \mathrm{~nm}$, Au thickness $d_{3}=20 \mathrm{~nm}, \mathrm{hBN}$ thickness $d_{4}=131 \mathrm{~nm}$, graphene layer $N=1$.

After setting the structural parameters, the dependence of the sensitivity of the GH shift on the refractive index $n_{s}$ of the sensing layer will be discussed in Figure 6 . When the hBN thickness is $131 \mathrm{~nm}$ and the Fermi energy is $0.2 \mathrm{eV}$, as shown in Figure 6a, the sensitivity curve of GH shift varies with refractive index $n_{S}$ of the sensing layer is plotted. When the refractive index changes between 1.2 1.35 RIU, the sensitivity curve changes drastically, and the sensitivity is higher in this range. Based on these two reasons, the variation of the refractive index of the sensing layer within this range is more convenient for detection in applications. At this time, the sensitivity can be achieved $1.76 \times 10^{4} \lambda / R I U$, while the refractive index is 1.252 . As mentioned before, the GH shift can be enhanced by increasing the hBN thickness. Therefore, the sensitivity curve is plotted in Figure $6 \mathrm{~b}$, and the sensitivity can reach values as high as $2.02 \times 10^{5} \lambda / R I U$ when the refractive index is 1.306. However, in Figure $6 \mathrm{~b}$, the range of sharp sensitivity varies is shifted to the right to $1.28-1.36$, and the sensitivity detection range is slightly reduced. It can be concluded that the sensitivity can be improved by magnifying the thickness of hBN, but the best detection range will shift to a large refractive index and the detection range of high sensitivity will be narrowed correspondingly. Therefore, we can select the appropriate thickness of $\mathrm{hBN}$ according to the predicted refractive index range in practical application, and then tune the Fermi energy to advance the sensitivity. The refractive index sensor based on GH shift interrogation mode has the advantages of simple structure, high-sensitivity, and tunability, which are of great significance for the design of infrared band precise measurement or optical sensor. 

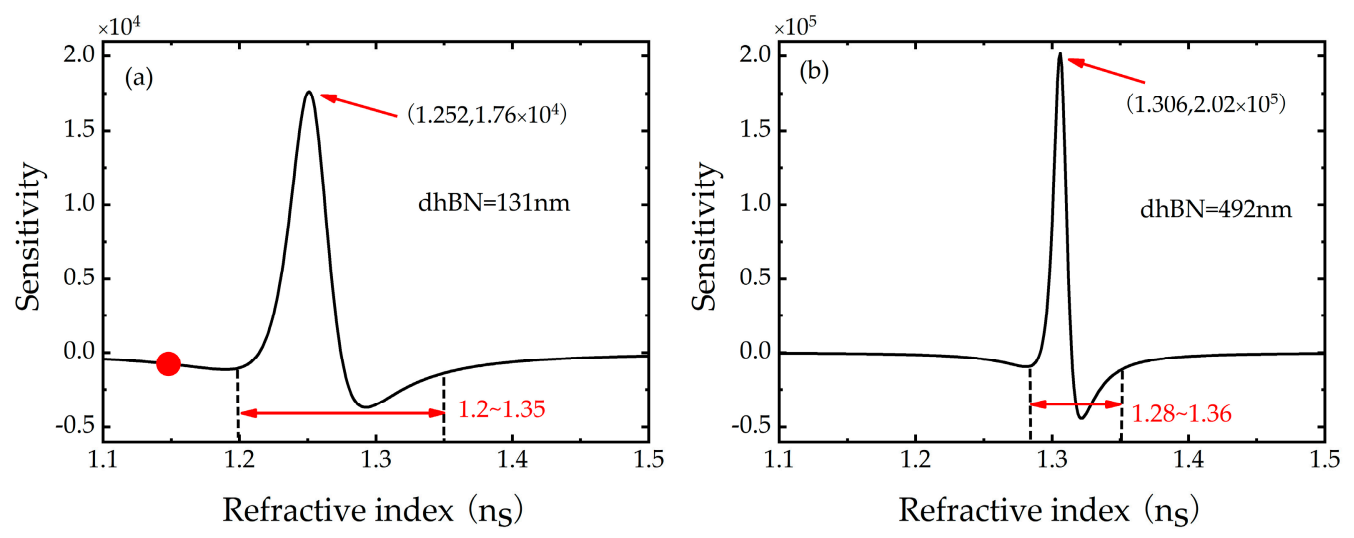

Figure 6. (a) Dependence of sensitivity of GH shift on refractive index ns of sensing layer when the hBN thickness is $131 \mathrm{~nm}$, (b) Dependence of sensitivity of GH shift on refractive index ns of sensing layer when the hBN thickness is $492 \mathrm{~nm}$, in which Fermi energy $E_{F}=0.2 \mathrm{eV}$, Ag thickness $d_{2}=15 \mathrm{~nm}$, Au thickness $d_{3}=20 \mathrm{~nm}$, graphene layer $N=1$.

\section{Conclusions}

In summary, we propose a bimetal structure based on graphene-hBN to enhance the GH shift in the infrared band and design a sensitivity-tunable refractive index sensor based on the GH shift. The results of numerical simulation show that when the Fermi energy is $0.2 \mathrm{eV}$, a large $\mathrm{GH}$ shift can be obtained in monolayer graphene. In addition, when the thickness of gold layer is $20 \mathrm{~nm}$, silver layer is $15 \mathrm{~nm}$, and the thickness of hBN is $131 \mathrm{~nm}$, the sensitivity of the sensor can reach $1.76 \times 10^{4} \lambda / R I U$. Also, the sensitivity of the sensor can reach $2.02 \times 10^{5} \lambda / R I U$ when the thickness of $\mathrm{hBN}$ is $492 \mathrm{~nm}$, but the sensor can only be used to detect samples with high refractive index. Therefore, the thickness of hBN should be determined according to the refractive index of the sample. These results can provide a theoretical basis for the design of refractive index sensor with high sensitivity and accuracy in infrared band.

Author Contributions: Z.L., F.L., L.J. and W.L. performed the simulation experiments. Z.L., Z.Z. and F.L. wrote the paper. All authors have read and agreed to the published version of the manuscript.

Funding: This research was funded by National Natural Science Foundation of China, grant number 61905076, Hunan Provincial Natural Science Foundation of China, grant number 2019JJ50373 and The Science and Technology Innovation Program of Hunan Province, grant number 2020RC1003.

Institutional Review Board Statement: Informed consent was obtained from all subjects involved in the study.

Conflicts of Interest: The authors declare no conflict of interest.

\section{References}

1. Goos, F.; Hänchen, H. Einneuer und fundamentaler Versuchzur Totalreflexion. Ann. Phys. 1947, 436, 333-346. [CrossRef]

2. Sakata, T.; Togo, H.; Shimokawa, F. Reflection-type $2 \times 2$ optical waveguide switch using the Goos-Hänchen shift effect. Appl. Phys. Lett. 2000, 76, 2841-2843. [CrossRef]

3. Tang, M.; Ran, M.; Chen, F.; Wang, X.; Li, H.; Chen, X.; Cao, Z. Narrow band optical filter using Goos-Hänchen shift in a cascaded waveguide structure. Opt. Laser Technol. 2014, 55, 42-45. [CrossRef]

4. Wang, X.; Yin, C.; Sun, J.; Li, H.; Wang, Y.; Ran, M.; Cao, Z. High-sensitivity temperature sensor using the ultrahigh order mode-enhanced Goos-Hänchen effect. Opt. Express 2013, 21, 13380-13385. [CrossRef] [PubMed]

5. Wang, L.; Zhu, S. Large positive and negative Goos-Hänchen shifts from a weakly absorbing left-handed slab. J. Appl. Phys. 2005, 98, 043522. [CrossRef]

6. Liu, F.; Xu, J.; Song, G.; Yang, Y. Goos-Hänchen and Imbert-Fedorov shifts at the interface of ordinary dielectric and topological insulator. JOSA B 2013, 30, 1167-1172. [CrossRef]

7. Wang, L.; Chen, H.; Zhu, S. Large negative Goos-Hänchen shift from a weakly absorbing dielectric slab. Opt. Lett. 2005, 30, 2936-2938. [CrossRef]

8. Miri, M.; Naqavi, A.; Khavasi, A.; Mehrany, K.; Khorasani, S.; Rashidian, B. Geometrical approach in physical understanding of the Goos-Haenchen shift in one-and two-dimensional periodic structures. Opt. Lett. 2008, 33, 2940-2942. [CrossRef] 
9. He, J.; Yi, J.; He, S. Giant negative Goos-Hänchen shifts for a photonic crystal with a negative effective index. Opt. Express 2006, 14, 3024-3029. [CrossRef]

10. Nie, Y.; Li, Y.; Wu, Z.; Wang, X.; Yuan, W.; Sang, M. Detection of chemical vapor with high sensitivity by using the symmetrical metal-cladding waveguide-enhanced Goos-Hänchen shift. Opt. Express 2014, 22, 8943-8948. [CrossRef]

11. Wang, Q.; Jiang, X.; Wang, X.; Dai, X.; Xiang, Y. Enhancing photonic spin Hall effect in the surface plasmon resonance structure covered by the graphene-MoS2 heterostructure. IEEE Photonics J. 2017, 9, 1-10. [CrossRef]

12. Zheng, Z.; Lu, F.; Jiang, L.; Jin, X.; Dai, X.; Xiang, Y. Enhanced and controllable Goos-Hänchen shift with graphene surface plasmon in the terahertz regime. Opt. Commun. 2019, 452, 227-232. [CrossRef]

13. Jiang, L.; Wang, Q.; Xiang, Y.; Dai, X.; Wen, S. Electrically Tunable Goos-Hänchen shift of light beam reflected from a graphene-ondielectric surface. IEEE Photonics J. 2013, 5, 6500108. [CrossRef]

14. Tang, T.; Li, J.; Zhu, M.; Luo, L.; Yao, J.; Li, N.; Zhang, P. Realization of tunable Goos-Hänchen effect with magneto-optical effect in graphene. Carbon 2018, 135, 29-34. [CrossRef]

15. Zhou, X.; Liu, S.; Ding, Y.; Min, L.; Ming, L. Precise control of positive and negative Goos-Hänchen shifts in graphene. Carbon 2019, 149, 604-608. [CrossRef]

16. You, Q.; Shan, Y.; Gan, S.; Zhao, Y.; Dai, X.; Xiang, Y. Giant and controllable Goos-Hänchen shifts based on surface plasmon resonance with graphene-MoS2 heterostructure. Opt. Mater. Express 2018, 8, 3036-3048. [CrossRef]

17. Lin, Z.; Jiang, L.; Wu, L.; Guo, J.; Dai, X.; Xiang, Y.; Fan, D. Tuning and sensitivity enhancement of surface plasmon resonance biosensor with graphene covered Au-MoS 2-Au films. IEEE Photonics J. 2016, 8, 1-8. [CrossRef]

18. Shushama, K.N.; Rana, M.M.; Inum, R.; Hossain, M.B. Sensitivity enhancement of graphene coated surface plasmon resonance biosensor. Opt. Quant. Electron. 2017, 49, 381-394. [CrossRef]

19. Fouad, S.; Sabri, N.; Jamal, Z.A.Z.; Poopalan, P. Enhanced sensitivity of surface plasmon resonance sensor based on bilayers of silver-barium titanate. J. Nano Electron. Phys. 2016, 8, 04085. [CrossRef]

20. Kumar, R.; Kushwaha, A.S.; Srivastava, M.; Mishra, H.; Srivastava, S.K. Enhancement in sensitivity of graphene-based zinc oxide assisted bimetallic surface plasmon resonance (SPR) biosensor. Appl. Phys. 2018, 124, 235-245. [CrossRef]

21. Dhibi, A.; Sassi, I.; Oumezzine, M. Surface plasmon resonance sensor based on bimetallic alloys grating. Indian J. Phys. 2016, 90, 125-130. [CrossRef]

22. Tong, J.; Jiang, L.; Chen, H.; Wang, Y.; Yong, K.; Forsberg, E.; He, S. Graphene-bimetal plasmonic platform for ultra-sensitive biosensing. Opt. Commun. 2018, 410,817-823. [CrossRef]

23. Liu, L.; Wang, M.; Jiao, L.; Wu, T.; Xia, F.; Liu, M.; Kong, W.; Dong, L.; Yun, M. Sensitivity enhancement of a graphene-barium titanate-based surface plasmon resonance biosensor with an $\mathrm{Ag}-\mathrm{Au}$ bimetallic structure in the visible region. JOSA B 2019, 36, 1108-1116. [CrossRef]

24. Wang, X.; Liang, Y.; Wu, L.; Guo, J.; Dai, X.; Xiang, Y. Multi-channel perfect absorber based on a one-dimensional topological photonic crystal heterostructure with graphene. Opt. Lett. 2018, 43, 4256-4259. [CrossRef]

25. Zheng, Z.; Lu, F.; Dai, X. Tunable reflected group delay from the graphene/hBN heterostructure at infrared frequencies. Results Phys. 2019, 15, 102681. [CrossRef]

26. Wu, J.; Jiang, L.; Guo, J.; Dai, X.; Xiang, Y.; Wen, S. Tunable perfect absorption at infrared frequencies by a graphene-hBN hyper crystal. Opt. Express 2016, 24, 17103-17114. [CrossRef]

27. Shi, K.; Liao, R.; Cao, G.; Bao, F.; He, S. Enhancing thermal radiation by graphene-assisted hBN/SiO 2 hybrid structures at the nanoscale. Opt. Express 2018, 26, 591-601. [CrossRef]

28. Raether, H. Surface plasmons on smooth and rough surfaces and on gratings. Springer Tracts Mod. Phys. 1988, 111, 1-3.

29. Dai, S.; Ma, Q.; Liu, M.; Andersen, T.; Fei, Z.; Goldflam, M.D.; Wagner, M.; Watanabe, K.; Taniguchi, T.; Thiemens, M.; et al. Graphene on hexagonal boron nitride as a tunable hyperbolic metamaterial. Nat. Nanotechnol. 2015, 10, 682-686. [CrossRef]

30. Chen, P.; Alu, A. Atomically thin surface cloak using graphene monolayers. ACS Nano 2011, 5, 5855-5863. [CrossRef] [PubMed] 